\title{
Is mTOR involved in the mechanisms of the fast-acting antidepressant effects of AMPA receptor agonists?
}

\author{
Yimin Hu $\cdot$ Xiangliu Liu $\cdot$ Jinchun Shen
}

Received: 19 July 2012/ Accepted: 25 September 2012/Published online: 11 October 2012

(C) Springer-Verlag Wien 2012

We read with great interest the article "The involvement of AMPA-ERK1/2-BDNF pathway in the mechanism of new antidepressant action of prokinetic meranzin hydrate", in which the authors concluded that AMPA receptors were involved in the mechanism of the antidepressant effects of prokinetic meranzin hydrate (Xie et al. 2012). We appreciate the author's excellent perspectives and want to raise a hypothesis regarding the mechanisms of $\alpha$-amino3-hydroxy-5-methyl-4-isoxazolepropionic acid (AMPA) receptor agonists exerting fast-acting antidepressant effects.

A series of studies have demonstrated that AMPA receptor agonist such as LY392098 show fast-acting antidepressant effects in the rodent animal models ( $\mathrm{Li}$ et al. 2001). Moreover, Maeng et al. (2008) have observed that pretreatment with AMPA receptor antagonist NBQX can significantly reduce the ketamine's antidepressant effects. The ketamine-induced increase in glutamate release preferentially favors AMPA receptors over NMDA receptors because the latter have been occupied by ketamine, therefore the net antidepressant effect of ketamine is increased by glutamatergic throughput (Machado-Vieira et al. 2009). Collectively, these studies have indicated that AMPA receptors are essential for ketamine to exert its antidepressant effects (Duman and Voleti 2012). Recently, an innovative study conducted by Li et al. (2010) showing that mammalian target of rapamycin (mTOR) activation in prefrontal cortex is involved in the ketamine exerting fastacting antidepressant effects. Thus, we hypothesized that
AMPA receptor agonists exerting their antidepressant effects are potentially via up-regulating Akt and ultimately activating mTOR. Future studies are needed to verify the hypothesis and enrich this field knowledge.

Conflict of interest We declare no potential conflicts of interest.

\section{References}

Duman RS, Voleti B (2012) Signaling pathways underlying the pathophysiology and treatment of depression: novel mechanisms for rapid-acting agents. Trends Neurosci 35:47-56

Li X, Tizzano JP, Griffey K, Clay M, Lindstrom T, Skolnick P (2001) Antidepressant-like actions of an AMPA receptor potentiator (LY392098). Neuropharmacology 40:1028-1033

Li N, Lee B, Liu RJ et al (2010) mTOR-dependent synapse formation underlies the rapid antidepressant effects of NMDA antagonists. Science 329:959-964

Machado-Vieira R, Salvadore G, Diazgranados N, Zarate CA Jr (2009) Ketamine and the next generation of antidepressants with a rapid onset of action. Pharmacol Ther 123:143-150

Maeng S, Zarate CA Jr, Du J et al (2008) Cellular mechanisms underlying the antidepressant effects of ketamine: role of alphaamino-3-hydroxy-5-methylisoxazole-4-propionic acid receptors. Biol Psychiatry 63:349-352

Xie Y, Huang X, Hu S, Zhang Y, Wang Y, Qiu X, Ren P, Fan R, Zhang C, Xie W, Ji H, He J, Chen X, Xie L, Liu Z, Zhou H (2012) The involvement of AMPA-ERK1/2-BDNF pathway in the mechanism of new antidepressant action of prokinetic meranzin hydrate. Amino Acids. doi:10.1007/s00726-012$1347-2$
Y. Hu $\cdot$ X. Liu $\cdot$ J. Shen $(\varangle)$

Department of Anesthesiology, Jinling Hospital,

School of Medicine, Nanjing University,

No. 305 East Zhongshan Road, Nanjing 210002, China

e-mail: yyshen0203@163.com 